\title{
Hacking the code for reverse-aging
}

\begin{abstract}
Scientists of Prof. Cai's Lab of Albert Einstein Medical College in N.Y.N.Y. have broken the code of reverse aging of lab mice. They have identified micro-RNA generated from Hypothalamus stem cells can reverse the aging of lab mouse. At this critical junction, we need to do the same to large scale human subjects following the NIH Gold Standard. Instead of relying on Venture Capitalists (VC) investment, a large amount of federal public funding may be required to maintain the neutrality of the knowledge for Homosapiens.
\end{abstract}

Volume I Issue 4 - 2017

\begin{abstract}
Harold Szu
Department of Biomedical Engineering, The Catholic University, USA

Correspondence: Harold Szu, Department of Biomedical Engineering, The Catholic University, Wash DC, USA, Email szuharoldh@gmail.com
\end{abstract}

\section{Introduction}

World War II Baby-Boomers are getting old about 40 millions of them. They are facing aging-related disorders: e.g. Type-II Diabetics, Heart Attacks, and Strokes as well as some forms of Cancers. Nevertheless, their experience and judgment is a terrible thing to be wasted. Scientists at Google, CALICO (CA Life Co), National Institute of Aging (NIA; HHS/NIH) all believe that mankind is on the brink to be, so-to-speak, rocketed to, besides the moon, the "immortality promising land," in a sufficient escape velocity. This is microscopically hacking the code of reverse aging at the cellular level. Besides the Nobel, the Kyoto, the Templeton Prizes, there is a dedicated foundation initiated by Dr. Joon Yun, who is personally sponsoring the \$ million Palo Alto Longevity Prize through the private foundation Palo Alto Institute. It is a life science competition dedicated to ending aging, since Sept 9, 2004, Longevity Demonstration Prize ends September 9, 2018 to hack the code of life and cure aging. (Parts in Homeostasis, hearts beats etc. \& Parts in Longevity Demonstration). Time Magazine has featured that babies of the Year born after 2015 can live 142years old all the way to 2157, (Figure 1). The 2009 Nobel Laureates in Physiology \& Medicine, Prof. Katherine Blackburn at UC Berkeley \& Carol W. Greider (former Ph D Student) and Dr. Jack W. Szostak (Harvard) discovered the telomerase enzyme has an intrinsic clock of L. Hayflick (1965) 50turns, before the Apoptosis gene is set into a programming death. Moreover, the 2012 Nobel Laureate in Physiology \& Medicine Dr. Shinya Yamanake of UCSF and now at Kyoto has identified 4 specific genes called induced Pluripotent Stem (iPS) named by him which can rewind back the Clock at the embryonic state. Recently, Dr. Dengsheng Cai of Albert Einstein Medical College (AEMC) has cracked the code of reverse aging--- "It's all in your head, and the secrete lies in the micro-RNA in the Hypothalamus that has been known to control the metabolism of the time clock, growth maturity.

The micro-RNA (miRNA) is a short, non-coding RNA, It is not the messenger-RNA (mRNA) for DNA synthesis; rather, the miDNA prevents mRNAs synthesis into DNA, by binding on mRNA to reproduce aged protein. This fact has been done in the NIH Gold Standard fashion (Double Blind (DB), Negative Control (NC) \& Sufficient Statistics (SS)) to the lab mice successively; human subject studies are desperately needed. At this junction of promising cross road, we need to usher in unprecedented multidisciplinary research on the Homosapiens. We plead to the federal agency \$B funding to conduct large-scale human studies that will be overseen by HHS/ NIH with the Gold Standard (DB, NC,SS). The federally funded work without being limited by private investment IP Right Restriction, e.g. Silicon Valley VC, so that to live longer become a basic human right rather only for affordable rich people.

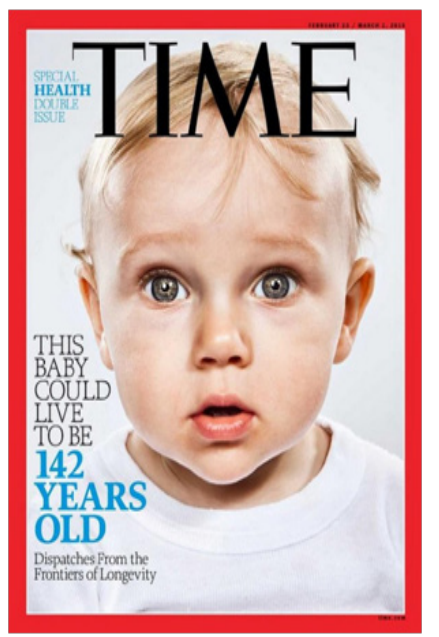

Figure I Children born after 2015 can live to be 142 years old by 2157.

From the irreversible thermodynamics point of view, the incessant inter-molecular collision mixing will increase the "degree of uniformity," measured by the Boltzmann Entropy S, leading to the heat death.

\section{$\Delta \mathrm{S}>0$}

However, the irreversible thermodynamics heat death did not prevent an open dynamic system to rejuvenate itself. For example, one can shuffle uniformly covered snow, at a maximum entropy, out of highway to make the highway passable.

$$
\Delta \mathrm{S} \leq 0
$$

\section{The basics of cellular biology}

In order to help the public to influence the congressional representatives, the general public needs to be educated about the reverse aging phenomenology. Herewith, we provide the necessary basics. We begin with the evolution of our ancestor at singe cells level which have hijacked the Mitochondria bacterial as the power house based on Adenosine triphosphate (ATP) to become multiple cell organisms to achieve survival functionality. 


\section{Symbiotic evolution partner mitochondria bacterial as efficient power house}

Our multiple cells have evolved billions of years from molecular level by taking into account the molecular cellular dynamics, due to our power house co-evolution partner: Mitochondria bacteria's (Figure 1A) (Figure 1B). Then, single cells become possible in the ocean environment to hijack other single cells Mitochondria (Mt) bacteria's to aggregate into a cluster in 3D structure. The Mitochondria has its own DNA in the circular genomes that were engulfed during metazoans period by the early ancestors of today's eukaryotic cells. Human mtDNA is inherited from the mother, as an egg contains on average 200,000mtDNA molecules, whereas a healthy human sperm was reported to contain on average 5mtDNA molecules. In most multicellular organisms, the mtDNA - or Mitogenome - is organized as a circular, covalently closed, double-stranded DNA. Each can provide a much higher ATP energy production, so that a cluster of multiple cells becomes possible to achieve different survival functionality. Eventually, the organism migrated to land and paddling fins became leggy animals.

\section{Genome versus phenome, namely DNA versus epige-} netic( methylation \& histone)

What is gene DNA?

Figure 2 Nobel Laureates Crick \& Watson determined the double helix of DeoxyriboNucleic Acid (DNA) which is a macro-molecule made of different repetition of alternating sugar-phosphate backbone: A(Adenine)- $\mathrm{T}$ (Thymine) \& $\mathrm{C}($ Cytosine $)-\mathrm{G}($ Guanine $)$ pairs (in hydrogen-co-valance-bound). Human has 3 billion pairs of A-T C-G codons of DNA packed in 23 pairs in a total of 46 chromosomes unwinded linearly in $3 \mathrm{~m}$ long.

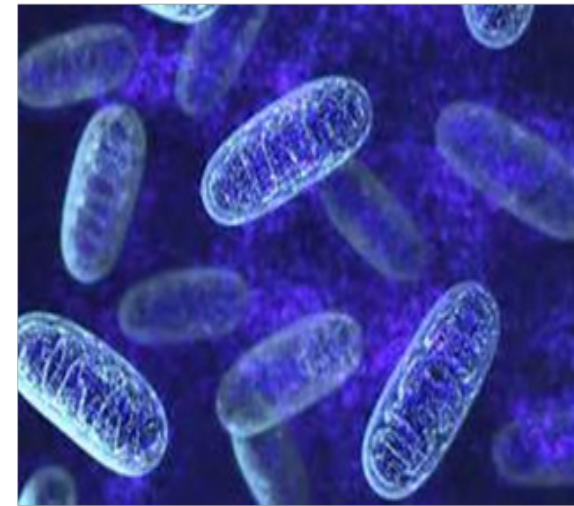

Figure I(A) Mitochondria Bacteria's.

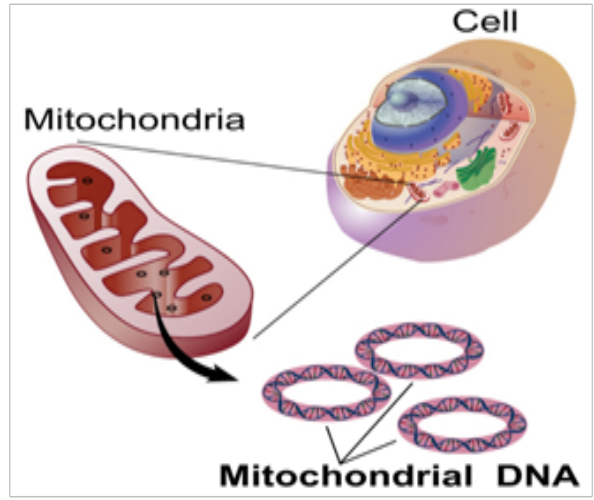

Figure I(B) A human mitochondrial contains $37 \mathrm{mtDNA}$ genes.
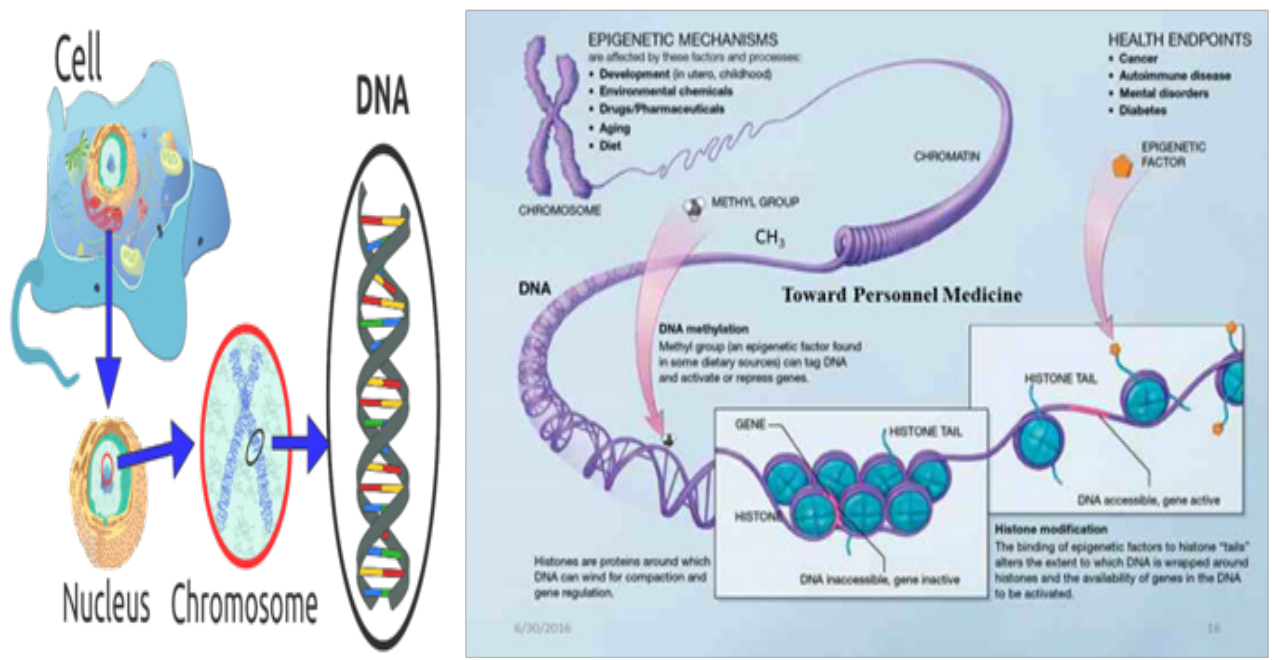

Figure 2

(A) Nucleus Chromosome DeoxyriboNucleic Acid (DNA);

(B) The Phenome is the outside explicit genes when the folding of linear DNA into 3D forms: Chromosomes. This folding is differentiated by attached Methylation $(\mathrm{CH}-3)$ tails what should be wound outside the Histone spooling.

What is epigenetics (Greek: outside)?

The epigenetic phoneme typing is mediated by the Methylation $\left(\mathrm{CH}_{3}\right)$ biomarkers, and Histone Spooling of gene-expression or gene-suppression. Epic (Greek: Outside) Genetics that is Phoneme environmental control of Genome through the biomarker $\mathrm{CH}_{3}$ Methylation as Histone spooling to make segments of genes expressive revealing or suppressive hidden. 


\section{What is the micro-RNA?}

It is a short non-coding RNA that regulates human genes and binding messenger-RNA prevents their translation into proteins, e.g. aged protein (Figure 3). The reverse aging has been investigated in human by Dr. Nir Barzilai of Albert Einstein College of Medicine
(AECM) by the Bronx, New York conducted a double blind control study beginning in 2015 of roughly 3000 elderly people; half would get a placebo and half would get an old (indeed, ancient) drug for type 2 diabetes called Metformin, which has been shown to modify aging in some animal studies.

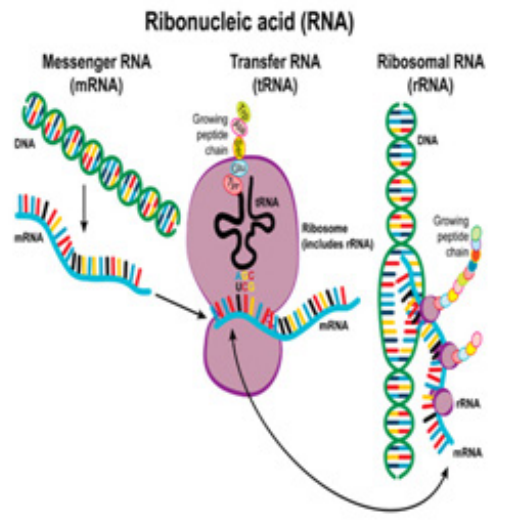

\section{Micro RNAs (miRNAs)}

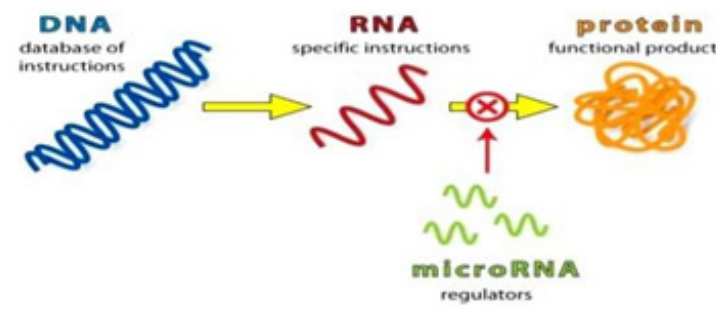

microRNAs, short non-coding RNAs present in all living organisms, have been shown to regulate the expression of at least half of all human genes. These single-stranded RNAs exert their regulatory action
binding messenger RNAs and preventing their translation into proteins.
Hypothalamus:

time clock, pituitary

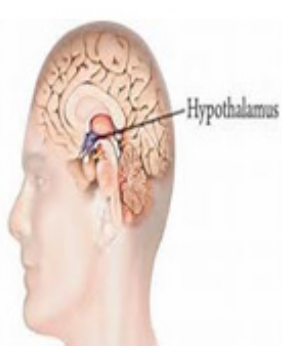

Figure 3

(A) Three kinks of single strand RNA: Messenger RNA (mRNA); Transfer RNA (tRNA); and Ribosomal RNA (rRNA);

(B) To control of these RNA's for synthesis, there are regulator known as micro-RNA(miRNA), which are short non-coding RNA's when bonded with coding RNA preventing these longer coding RNA's translated into proteins, say aged one. This is the basic mechanism

(C) How Hypothalamus keeping miRNA to rejuvenate Homosapiens.

\section{Hacking the code of reverse aging}

Microscopically, Dr. Dengsheng Cai studied aging from AECM Molecular Pharmaceutical Researchers on Reverse Aging in mice with stem cells ${ }^{1}$ (VOA's Kevin Enochs reports, August 13, 2017). Dr. Dongsheng Cai from the Molecular Pharmacology of AECM has invigorated mice in an area of the brain called the Hypothalamus which is known to control the growth \& reproduction metabolism of mice and published in Nature Magazine. He conducted a Gold Standard Control Study (Double-Blind, Negative Control, Sufficient Statistics) Stem cells of micro-RNA that inhibitory to certain protein formation the Hypothalamus setting the body's clock:

a. Aging mice injected at Hypothalamus the Stem-cell all became younger which requires DB;

b. Younger mice have removed the micro-RNA stem-cell of Hypothalamus became aged rapidly which require $\mathrm{NC}$;

c. Older mice injected micro-RNA Stem cells to ascertain about the micro-RNA with SS. The Scientists of Dr. Cai Lab of AECM in New York have found a way to turn back that clock, at least in mice. Hypothermia modulates circadian clock gene expression in lizard peripheral tissues; Hyperthermia, hyperthyroidism, and time judgment. Researchers on Reverse Aging in Mice with Stem Cells (VOA's Kevin Enochs reports, August 13, 2017).

Dr. Dongsheng Cai from the Molecular Pharmacology of AECM in New York has invigorated mice in an area of the brain called the Hypothalamus which is known to control the growth \& reproduction metabolism of mice and published in Science Magazine. He conducted Gold Standard Control Study Stem cells of the Hypothalamus setting the body's metabolic clock (Figure 4). a. Longevity may be due to Nonstop Production of Telomerase Enzyme in Figure 5.

b. P. Mattson's (NIH/NIA Dir.) "Dietary (Calorie) Restriction Normalizes Glucose Metabolism and Brain-Derived Neurotrophic Factor Levels, Slows Disease Progression and Increases Survival in Huntington Mutant Mice" PNAS Feb. 10, 2003;

c. Joel Furhman, "Eat to Live" National Best Sell 2004.

d. Shinya Yamanaka 4 Genes: induced Pluripotent Stem cells (also known as iPS cells or iPSCs) are a type of Pluripotent stem cell that can be generated directly from adult cells., ${ }^{2,3}$ The iPSC technology was pioneered by Shinya Yamanaka's (UCSF) lab in Kyoto, Japan, who showed in 2006 that the introduction of four specific genes encoding transcription factors could convert adult cells into Pluripotent stem cells. He was awarded the 2012 Nobel Prize along with Sir John Gurdon "for the discovery that mature cells can be reprogrammed to become Pluripotent." The Pluripotent stem cells hold great promise in the field of regenerative medicine. Because they can propagate indefinitely, as well as give rise to every other cell type in the body (such as neurons, heart, pancreatic, and liver cells), they represent a single source of cells that could be used to replace those lost to damage or disease. The most well-known type of Pluripotent stem cell is the embryonic stem cell.

Shinya Yamanaka discovery that adult somatic cells can be reprogrammed into Pluripotent cells has had a profound effect on developmental and stem cell biology. By introducing the genes for four factors that turn genes on and off, he induced the skin cells of adult mice to become like embryonic stem cells. He focuses on ways to generate cells resembling embryonic stem cells by reprogramming somatic, or skin, cells to 4 induced Pluripotent Stem (iPS) Genes of mice \& human (Figure 5). 

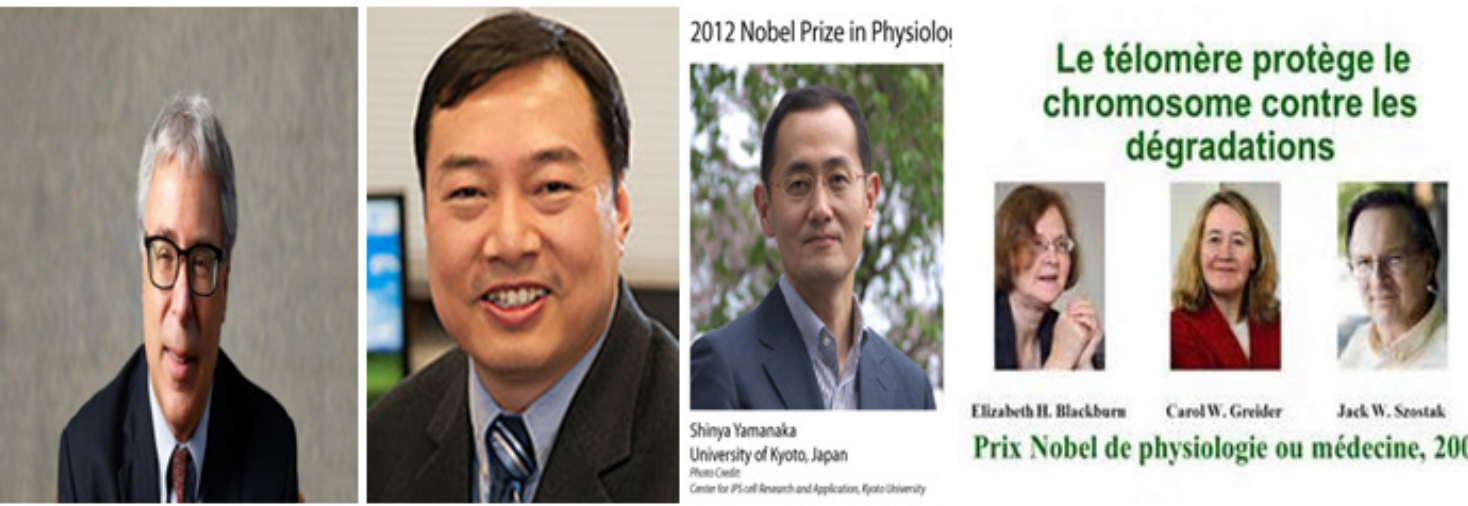

Figure 4

(A) Dr. Nir Barzilai reverse human aging taking type 2 diabetic drug Metformine 2015;

(B) Dr. Dengsheng Cai micro-RNA of Stems Cells at Hypothalamus control aging \& reverse aging 20I7.' (I) Aging mice injected at Hypothalamus miRNA Stem-cell all became younger; (2) Younger mice have removed the stem-cell of Hypothalamus became aged rapidly; (3) older mice injected micro-RNA Stem cells to ascertain about the micro-RNA from the AECM in New York may have found a way to turn back the clock in mice.

(C) The Nobel Prize in Physiology or Medicine 2009 Dr. Elizabeth Blackburn *(UCSF), CarolW. Greider (former Ph. D. Student) and Dr.jackW. Szostak (Harvard) "Maintenance of chromosomes by telomeres and the enzyme telomerase."

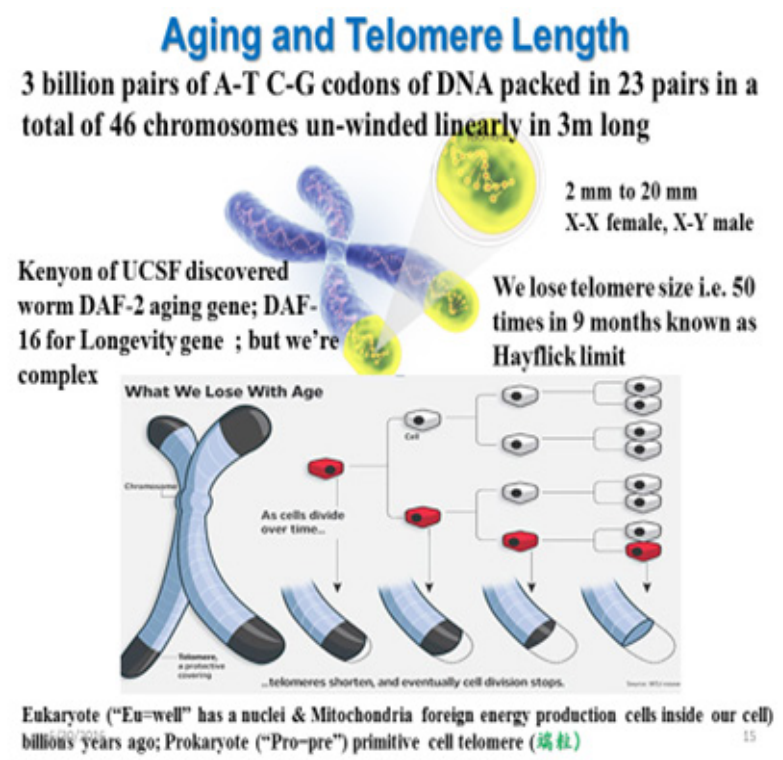

\section{Longevity May Attribute to Nonstop Production of Telomerase Enzyme \\ - Lobsters (non-stop gronth of telomerase keeping longevity) can live 150 yrs. 0n the contrary, mammas have high growth rate in embryonic and jwentle phases and no growth in adult and old phases)

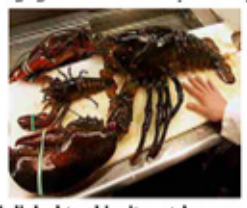 \\ "Longevity of lobsters is linked to ubiquitous telomerase expression," Klapper, et al U. Kiel, FEBS Leeters V. 439, pp.143-146 (1998)}

Galápagos giant tortoise of 190 years, Arctic Clam of 400 years
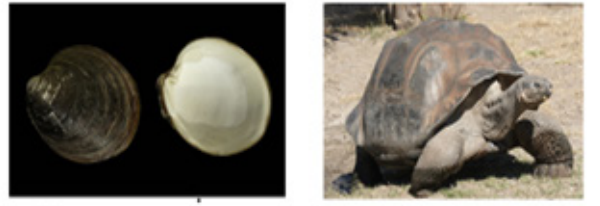

Figure 5

(A) Telomerase Enzyme controls the monomer knots near the end of Chromosomes known as L. Hayflick (1965) 50 turns. $^{8}$

(B) The longest living animal has constantly secreted Telomerase Enzyme.

\section{Conclusion: bio-computation}

DNA is like a hardware of computer language that carries most of the genetic instructions used in the growth, development, functioning, and reproduction of all known living organisms and many viruses. This is similar to genome \& phoneme (genetic DNA likes machine language hardware; epigenetic is like programming software). While Human Genome Program studied the hardware, European Epigenetic Programs studied the software. We have investigated many identical twins that their lifestyles may have influenced the Epigenetics that pass down to influence the next gen. We understand the disorder and the longevity better. Stem cells of microRNAs can set the
Hypothalamus, mice's clock reversing the aging, which remains to be demonstrated for a human with no string attached federal funding. "In God We Trust, All the Rest Show Data". A common sense of keeping the longevity of 142years old might be summarized as: "Eat Less, ${ }^{4,5}$ Exercise More, Sleep Tight, ${ }^{6-9} \&$ Be Happy," in 8 words.

\section{Acknowledgments}

None.

\section{Conflicts of interest}

Author declares that there is no conflict of interest. 


\section{References}

1. Zhang Y, Kim M, Jia B, et al. Hypothalamic stem cells control aging speed partly through exosomal miRNAs. Nature. 2017;548(7665):52-57.

2. Yamanaka S. Elite and stochastic models for induced pluripotent stem cell generation. Nature. 2009;460(7251):49-52.

3. Okita K, Ichisaka T, Yamanaka S. Generation of germline-competent induced pluripotent stem cells. Nature. 2007;448(7151):313-317.

4. Duan W, Guo Z, Jiang H, et al. Dietary (Calorie) Restriction Normalizes Glucose Metabolism and Brain-Derived Neurotrophic Factor Levels, Slows Disease Progression and Increases Survival in Huntington Mutant Mice. Proc Natl Acad Sci USA. 2003;100(5):2911-2916.
5. Joel Fuhrman. Eat to Live. National Best Seller. 2004.

6. Nedergaard M, Goldman SA. Brain Drains. Sci Am. 2016;314(3):45-49.

7. Defronzo RA, Barzilai N, Simonson DC. Mechanism of Metformin Action in Obese and Lean Noninsulin-Dependent Diabetic Subjects. J Clin Endocrinol Metab. 1991;73(6):1294-1301.

8. Hayflick L. The limited in vitro lifetime of human diploid cell strains. Exp Cell Res. 1965;37(3):614-636.

9. Blackburn EH, Jack WS, Carol WG. Maintenance of chromosomes by telomeres and the enzyme telomerase. The Nobel Prize in Physiology or Medicine. 2009. p. 1-12. 


\section{Author Biography}

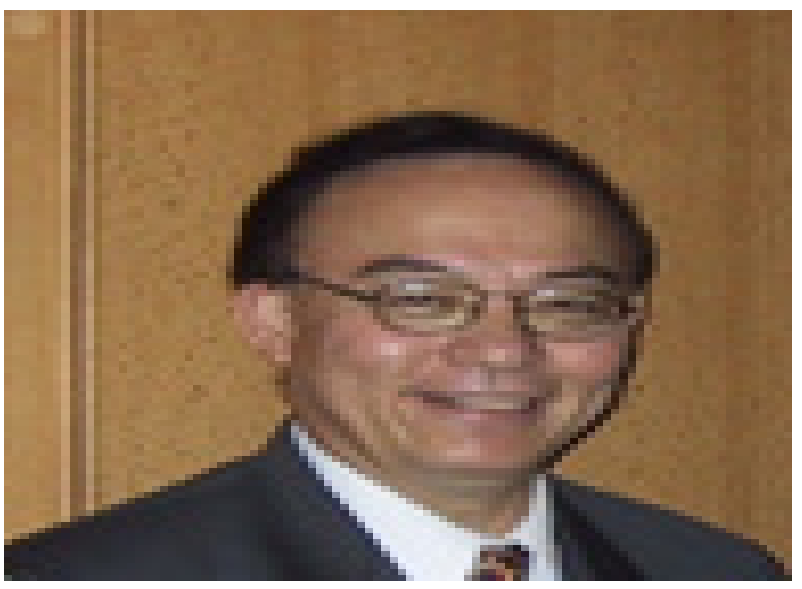

Dr. Harold HwaLing Szu has been a champion of Unsupervised Deep Learning Computational brain-style Natural Intelligence for 3 decades. He received the INNS D. Gabor Award in 1997 "for outstanding contribution to neural network applications in information sciences. He pioneered the implementations of fast simulated annealing search. He received the Eduardo R. Caianiello Award in
1999 from the Italy Academy for "elucidating and implementing a chaotic neural net as a dynamic realization for fuzzy logic membership function. Dr. Szu is a foreign academician of Russian Academy of Nonlinear Sciences for his interdisciplinary Physicist-Physiology to Learning (\#135, Jan 15, 1999, St. Petersburg). He is a Fellow of American. Institute Medicine \& Bio Engineering 2004 for passive spectrogram diagnoses of cancers; Fellow of IEEE $(\# 1075,1997)$ for bi-sensor fusion; Fellow of Optical Society America (1995) for adaptive wavelet; Fellow of International Optical Engineering (SPIE since 1994) for neural nets; Fellow of INNS (2010) for a founding secretary and treasurer and former president of INNS. Dr Szu has graduated from the Rockefeller University 1971, as thesis student of G. E. Uhlenbeck. He became a visiting member of Institute of Advanced Studies Princeton NJ, as well as a civil servant at NRL, NSWC, ONR, and then a senior scientist at Army Night Vision Electronic Sensory Directorate, Ft. Belvoir VA over 40 years. To pay back the community, he served as research professor at AMU, GWU, and CUA, in Wash DC. Besides 640 publications, over dozen US patents, numerous books \& journals (cf. researchgate.net/ profile/Harold_Szu2). Dr. Szu taught thesis students "lesson in creativity: editorial" (for individual with 4C principles and for a group by 10 rules) following a Royal Dutch tradition from Boltzmann, Ehrenfest, \& Uhlenbeck (Appl. Opt. 54 Aug. 10, 2015). He has guided over $17 \mathrm{PhD}$ thesis students. 